\title{
Nonlinear Optical Fibers, Their Properties, and Applications
}

\section{B. R. Ghimire}

\section{Journal of Nepal Physical Society}

Volume 6, Issue 2, December 2020

ISSN: 2392-473X (Print), 2738-9537 (Online)

\section{Editors:}

Dr. Binod Adhikari

Dr. Bhawani Joshi

Dr. Manoj Kumar Yadav

Dr. Krishna Rai

Dr. Rajendra Prasad Adhikari

Mr. Kiran Pudasainee

JNPS, 6 (2), 121-126 (2020)

DOI: http://doi.org/10.3126/jnphyssoc.v6i2.34867

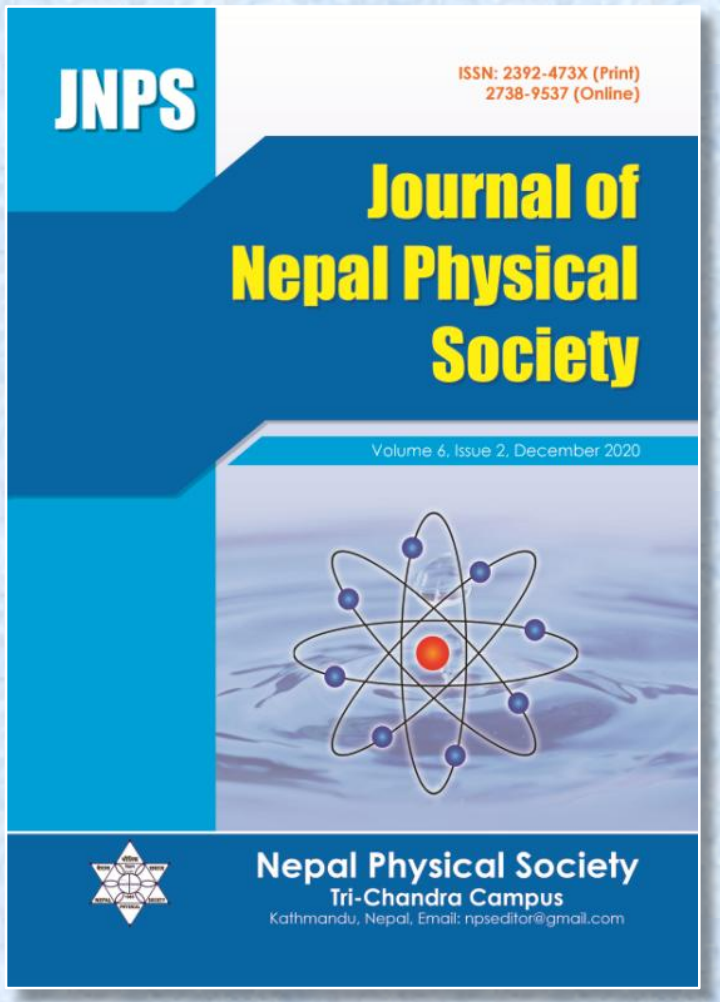

Published by:

Nepal Physical Society

P.O. Box: 2934

Tri-Chandra Campus

Kathmandu, Nepal

Email: npseditor@gmail.com 


\title{
Nonlinear Optical Fibers, Their Properties, and Applications
}

\author{
B. R. Ghimire \\ Central Department of Physics, T. U., Kirtipur, Kathmandu \\ Corresponding Email: balram.ghimire@cdp.tu.edu
}

Received: 23 October, 2020; Revised: 25 November, 2020; Accepted: 25 December, 2020

\begin{abstract}
Nonlinear optical properties and preparation of nonlinear optical fiber based on Bismuth oxide were described. Wavelength dependence of refractive index of ABH160 and B027 was studied and analyzed. Working and construction of optical ON-OFF switches, based on Bi-HNLF was reported. It was investigated that refractive index of ABH160 and Bo27 were found to be exponentially decaying as a function of wavelength. Absorption coefficient of ABH160 as a function of wavelength was found to be decaying exponentially. In optical fiber the total group velocity dispersion is divided into the material dispersion $D_{M}$ and waveguide dispersion $D_{W}$. Material dispersion and waveguide dispersion were calculated theoretically and a good agreement with the experimental result. The process of four wave mixing and consequences was reviewed.
\end{abstract}

Keywords: Optical fiber, Nonlinearity, Plasmon, refractive index, group velocity, harmonic generation, Susceptibility.

\section{INTRODUCTION}

Rapid development in the fields like optoelectronics, telecommunication information processing, optical computing and optical data storage has been proved by significant advances in nonlinear optical materials in the past couple of decades [1]. Optical fiber and photonics devices for communication, sensing and entertainment have become more and more important in daily life. With a growing demand for larger and faster nonlinearities, increased attention has been focused on artificial materials which can offer high nonlinearity in particular on those that will allow nonlinear optical devices to operate efficiently at relatively low power levels. Materials with large third-order nonlinear are of great interest currently, since the nonlinear refractive indices can be exploited for switching, waveguides, optical bistability, phase conjugation and other type of signal processing [2-4]. It was observed that surface Plasmon resonance enhancement in phase conjugation measurements of third order susceptibility $\chi^{(3)}$ for system containing either silver or gold spheres. Later on it was concluded that the nonlinear optical response in golddoped glass near the surface plasmon resonances is dominate by hot- electron and interband transition which are size- independent. The interest in nonlinear optical properties of quantum-confined semiconductor and metal crystalline is motivated and justified on both technological and fundamental grounds.

Optical glasses with large Kerr nonlinearities are good candidates to use in nonlinear optical devices because of their ultra fast response to the electric field. The glass with the large refractive index generally shows the large optical nonlinearity. It was reported that bismuth-silicate glasses exhibit significantly large optical nonlinearities and show ultra fast nonlinear optical response, since they contain no toxic elements and they are thermally and chemically stable, glasses containing bismuth have enough potential to be used as nonlinear optical medium in near future. Because of the high confinement of light and long interactive length, nonlinear optical fiber is one of the most attractive candidates for devices using nonlinear optical materials. As the silica fiber has high transparency and controllable chromatic dispersion, it is extensively used in nonlinear fiber optics. The search for applicable photonics material particularly glasses with high third order nonlinear optical susceptibility $\chi^{(3)}$ has proved important advances 
in this filed. The host glasses with modified matrix network have been developed which led to improve third order nonlinearity. Now a days two techniques z-scan and Kerr shutter method have been employed which allows direct measurement of the electronic time response as well as the magnitude of the nonlinearity. Unlike the Z- scan method, from the Kerr shuttle method one does not infer the sign of the nonlinearity nor the imaginary part that is nonlinear absorption of $\chi^{(3)}$. Therefore both techniques are complementary.

In this work special attention is given to the description of High Non-linearity of Optical Fibers and their applications. High nonlinearity $\mathrm{Bi}_{2} \mathrm{O}_{3}-$ based glass optical fiber Bi-NLF is reviewed and wavelength dependence of the refractive index of ABH160 and B027 materials will be studied [5]. Also the applications of Bi-NLF are reported. As an application of Bi-HNLF the construction and working of all-optical ON-OFF switch will be reported.

Now a day's Bi-NLF and Bi-HNF based fibers have been used in different field of our life. Such fibers are used for high repetition rate signal processing, broad-band light source, optical time division multiplexing, demultiplexing based cross phase modulation, four wave mixing-based wavelength conversion, super continuum light generation and pulse compression, optical limiting, and enhanced second order nonlinearities. Also in metrology and medical imaging and sensing such fibers have great use. Recently use of Bi-HNLF for all-optical ONOFF switching also reported.

The experimental setup for all-optical ON-OFF Switch, based on Bi-HNLF, described by K. K. Quresi et al has been shown in the fig-3 [6].

Nonlinear optical effect has been observed since $19^{\text {th }}$ century with Pockel's effect and Kerr effect. High fields associated with laser become available in the 1960s and gave it rise to way new nonlinear optical effect like second harmonic generation, third harmonic generation, stimulated Raman scattering and self-focusing. In nonlinear optics we are concerned with the effects that the light itself induces as it propagates through the medium. In nonlinear optics the light is deflected or delayed but its frequency or wavelength is unaltered [8].

Polarization $\tilde{P}(t)$ of a material system depends upon the strength $\tilde{E}(t)$ of the optical field. In the case of linear optics, the induced polarization depends linearly upon the electric field strength in a manner that can often be described by relation

$$
\tilde{P}(t)=\chi^{(1)} \tilde{E}(t)
$$

Where the constant of proportionality $\chi^{(1)}$ is known as linear susceptibility. In nonlinear optics, the optical response can be described by generalizing above equation by expressing the polarization $\tilde{P}(t)$ as a power series in the field strength as $\widetilde{E}(t)$

$$
\tilde{P}(t)=\chi^{(1)} \tilde{E}(t)+\chi^{(2)} \tilde{E}(t)^{2}+\chi^{(3)} \tilde{E}(t)^{3}+\ldots \ldots
$$

Therefore,

$$
\widetilde{P}(t)=\widetilde{P}^{(1)}(t)+\widetilde{P}^{(2)}(t)+\widetilde{P}^{(3)}(t)+\ldots \ldots \ldots \ldots \ldots
$$

The quantities $\chi^{(2)}$ and $\chi^{(3)}$ are known as second and third order nonlinear optical susceptibility, respectively. It has been observed that second order nonlinear optical interactions can occur only in non-Centro symmetric crystals (that is crystals that do not display inversion symmetry like liquid, gases, glasses amorphous solid). The third order nonlinear optical interactions can occur both for Centro symmetric and non-Centro symmetric media.

Since the third order contribution to the nonlinear polarization is given by

$$
\tilde{P}^{(3)}(t)=\chi^{(3)} \tilde{E}(t)^{3}
$$

For monochromatic field $\widetilde{E}(t)$,

$$
\begin{aligned}
\tilde{E}(t) & =\varepsilon \cos \omega t \text { and } \cos ^{3}(\omega t) \\
& =\frac{1}{4} \cos (\omega t)+\frac{3}{4} \cos (\omega t)
\end{aligned}
$$

Therefore

$$
\tilde{P}^{(3)}(t)=\frac{1}{4} \chi^{(3)} \varepsilon^{3} \cos (3 \omega t)+\frac{3}{4} \chi^{(3)} \varepsilon^{3} \cos (\omega t)
$$

The first term in equation (5) leads to process of third harmonic generation. In the third harmonic generation three photons of frequency $\omega$ are destroyed and one photon of frequency $3 \omega$ is created in each elementary event [8] as shown in figure 1 below: 


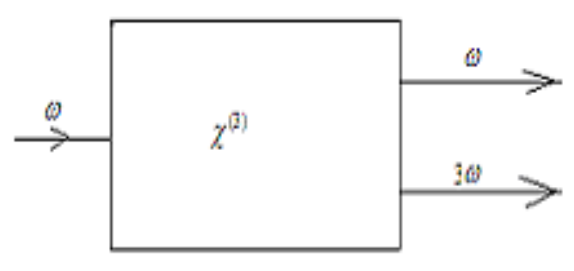

Fig--1(a)

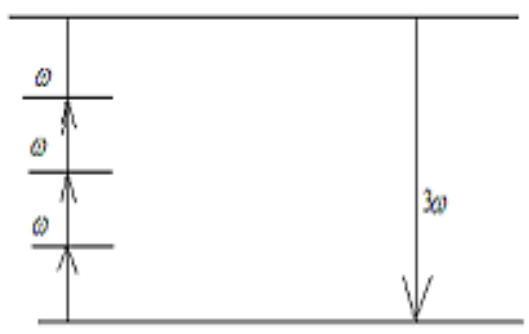

Fig--1(b)

Fig. 1: Third Harmonic Generation (a) Geometry interaction (b) Energy levels

But in general case,

$$
\widetilde{P}^{(3)}(t)=\chi^{(3)} \tilde{E}(t)^{3}
$$

With three components of $\tilde{E}(t)$ field

$$
\tilde{E}(t)=E_{1} e^{-i \omega_{1} t}+E_{2} e^{-\omega_{2} t}+E_{3} e^{-\omega_{3} t}+\text { c.c. }
$$

And

$$
\tilde{E}(t)=\sum_{n} p\left(\omega_{n}\right) e^{-i \omega_{n} t}
$$

\section{METHODOLOGY}

\section{Preparation and Optical Properties of Bi-NLF:}

The single mode fiber with mode-field diameter of $5.1 \mu \mathrm{m}$ was prepared by simply pouring a melted glass of $\mathrm{Bi}_{2} \mathrm{O}_{3}-\mathrm{SiO}_{2}$ having $78 \%$ of $\mathrm{Bi}_{2} \mathrm{O}_{3}$ in a mould to form a perform rod [5]. Mechanical, chemical and thermal durability of the fiber was sufficient for practical applications. Refractive indices of the core and cladding respectively were 2.02 and 2.01 at $1550 \mathrm{~nm}$. The fiber under test was $102.3 \mathrm{~cm}$ long and was fusion spliced with $\mathrm{SiO}_{2}$ at both ends. The splice loss was $0.48 \mathrm{db}$ at both ends and propagation loss was below $0.8 \mathrm{db} / \mathrm{m}$.

Hasegawa and coworkers reported that the linear and non-linear refractive index of glass containing $\mathrm{Bi}_{2} \mathrm{O}_{3}$ strongly depends on the $\mathrm{Bi}_{2} \mathrm{O}_{3}$ concentration. They explained experimental description of preparation of Bi-LNF in their paper in which $\mathrm{Bi}_{2} \mathrm{O}_{3}$ content glass $\mathrm{ABH} 160$ was prepared for the core medium B027 was developed for the cladding medium for the fiber [1]. The concentration of $\mathrm{Bi}_{2} \mathrm{O}_{3}$ in $\mathrm{ABH} 160$ and $\mathrm{B} 027$ were $65.5 \%$ and $55 \%$ respectively. The mixture was melted in a platinum crucible in a furnace in air at high temperature of $1000^{\circ} \mathrm{C}$ for 1 hour and then that was poured on the plate and then annealed. The glass plates were cut in the prism shape and were polished. By using the proper spectrometer, the wavelength dependence of refractive index was obtained at the wavelength range $502 \mathrm{~nm}$ to $1710 \mathrm{~nm}$. At the $1550 \mathrm{~nm}$ the mode field diameter was 1.97 and core diameter 1.61 micrometer and the refractive indices of ABH167 and B027 were 2.219 and 2.125 respectively. From four- wave mixing measurements using $102.3 \mathrm{~cm}$ long $\mathrm{Bi}$ - fiber, it was found that the Bi-fiber has a nonlinear coefficient as high as $\gamma=64.2 \mathrm{~W}^{-1} \mathrm{Km}^{-1}$ which is more than 20 times larger than $\gamma$ of $\mathrm{SiO}_{2}$ based $\mathrm{DSF}_{\mathrm{s}}$ not only this $\mathrm{Bi}$ - fiber reveals good mechanical, chemical and thermal stability but also the propagation loss is less than $0.8 \mathrm{~dB} / \mathrm{m}$ and it can be fusion, spliced to $\mathrm{SiO}_{2}$ fibers. Fig-5 represents the linear absorption coefficient and refractive index of ABH160. The refractive index $\mathrm{n}$ at $1550 \mathrm{~nm}$ is as high as 2.310 at $633 \mathrm{~nm}$ and 2.219 at $1550 \mathrm{~nm}$, induced by the fundamental absorption edge just below $600 \mathrm{~nm}$.

But no significant absorption was observed in nearinfrared region indicating that this glass can be used in telecommunication wavelength.

In the similar manner Chalcogenide glass fiber is other promising candidate for use as a nonlinear optical medium because of its high nonlinearity and interaction length that's why researchers trying to apply this material to all optical switching.

$$
n^{2}(\lambda)=1+\left(\frac{A_{1}}{\frac{1}{a_{1}^{2}}-\frac{1}{\lambda^{2}}}\right)-A_{2} \lambda^{2}
$$

This equation is called Sellmeier's equation where $a_{1} \quad$ is the resonant wavelength, $A_{j}$ is the Sellmeier's coefficient, the second term of the right side of this equation corresponds to the dispersion induced by the electronic transition which dominates the wavelength dependence of refractive index and the third term is the correction term.

First of all pump wavelength was fixed to $1560 \mathrm{~nm}$ and amplified by an $\mathrm{E}_{\mathrm{r}^{-}}$doped fiber amplifier and the 
pump wave was combined with the signal wave whose wavelength was tuned in the range over 1560 $\mathrm{nm}$ and launched on the Bi-fiber under test. The pump power incident on the Bi-fiber was estimated to be $23.8 \mathrm{dBm}$. The power ratio of the newly generated idler wave and the signal wave was determined from the output spectrum by an optical spectrum analyzer. The experimental setup for four-wave mixing is shown in fig-2 and the result is shown in fig- 6 . This shows the variation of the ratio of idler power to signal wave power with signal wavelength detuning from pump wavelength.

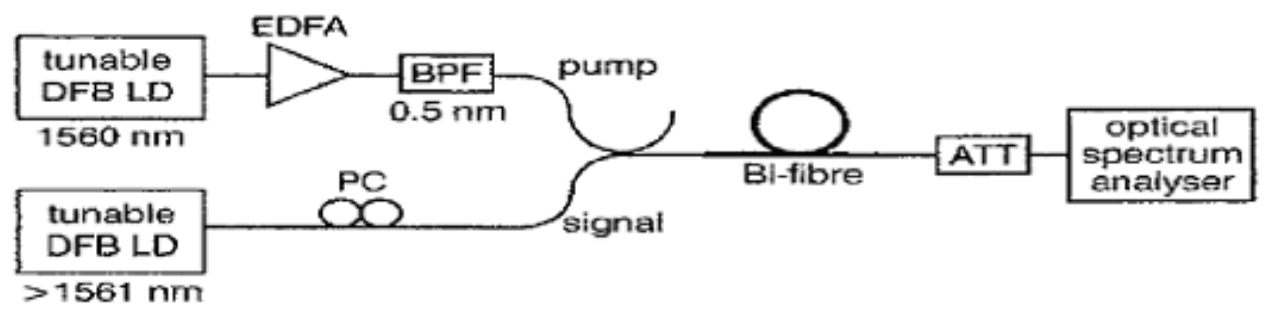

Fig. 2 : Experimental set up for four wave mixing [7].

The signal (at $1553.33 \mathrm{~nm}$ ) is generated by externally modulating a tunable laser (TL-1) with an electro-optic Lithium Niobate $\left(\mathrm{LiNbo}_{3}\right)$ modulator driven by a $10 \mathrm{~Gb} / \mathrm{s}$ pulse pattern generator (figure 2). The control signal (at $1554.13 \mathrm{~nm}$ ) was generated by external modulation of another tunable laser (TL-2). The wavelength separation between data signal and control signal was maintained $0.8 \mathrm{~nm}$ so that the idler wavelength can be placed $0.8 \mathrm{~nm}$ apart. The data and control signal are amplified separately to an average power of $300 \mathrm{~mW}$ by using EDFAs having maximum saturation power output power of $500 \mathrm{~mW}$. The PCs (polarization controllers) were used to check the states of polarization of both signals. Two signals are combined in an 8 channel DWDM multiplexer and then launched into $1.9 \mathrm{~m}$ of Bi-HNLF (figure $3)$. The mode field diameter and effective core area
$\left(\mathrm{A}_{\text {eff }}\right)$ of Bi-HNLF was estimated $2.1 \mu \mathrm{m}$ and 3.3 $\mu m^{2}$ respectively. After that the splicing process was done. If the direct fusion splicing would be done between Bi-HNLF and SMF-28 the splicing loss would be very large as the mismatch are 2.1 and $10.4 \mu \mathrm{m}$ for two fibers. Therefore to reduce splicing losses, two ends of Bi-HNLF are first connected to two segment of Ultra High silica fiber then was connected to silica fiber SMF-28. These fibers are fusion-spliced by arc discharge using conventional fusion splicing machine. The total loss in input of Bi-HNLF was $1.1 \mathrm{~dB}$ and the total loss output of Bi-HNLF was $2.6 \mathrm{~dB}$, therefore the propagation loss of the Bi-HNLF was $2.0 \mathrm{~dB} / \mathrm{m}$ at $1550 \mathrm{~nm}$. Thus the total loss in Bi-HNLF was $7.5 \mathrm{~dB}$ if the core and cladding material have refractive indices 2.22 and 2.13 respectively.

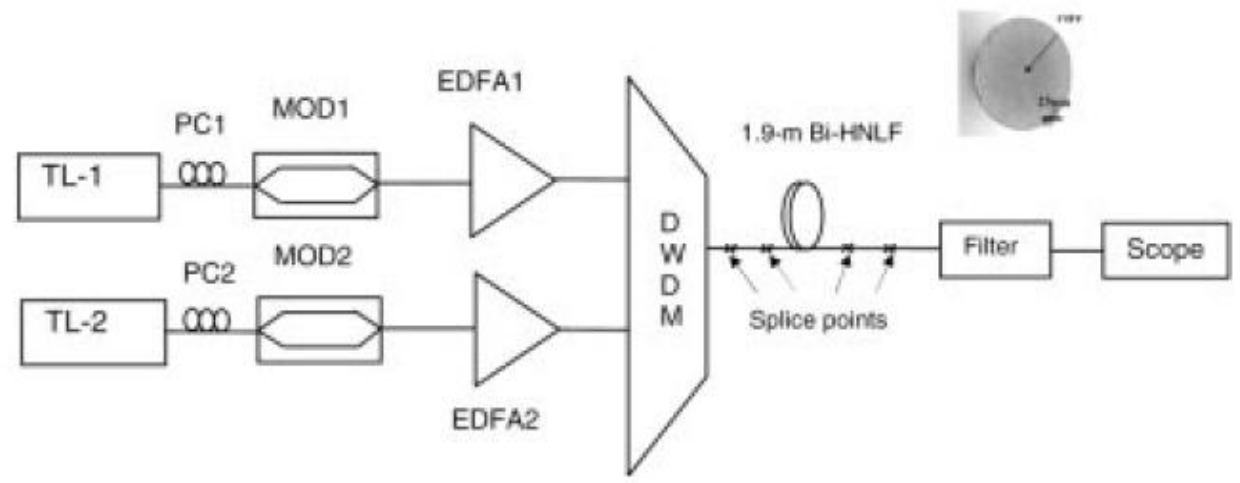

Fig. 3 : Experimental set up of all-optical Bi-HNLF based on-off switch [6]

\section{RESULT AND DISCUSSION}

The wavelength dependence of refractive index $n$ $(\lambda)$ of $\mathrm{Bi}_{2} \mathrm{O}_{3}$ based glasses are shown in fig- 4 which was obtained by plotting equation (9) analytically.
In fig-4 the solid line represents the wavelength dependence of refractive index of ABH160 and dotted line represents that of B027 respectively. This shows that refractive index almost decays exponentially with wavelength of the light. 


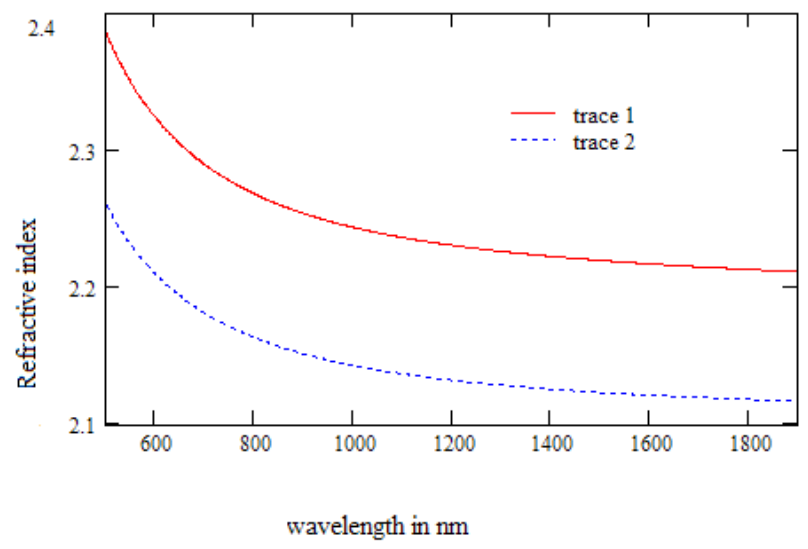

Fig. 4 : Refractive index as a function of wavelength of $\mathrm{ABH} 160$ and Bo27

The group velocity dispersion is an important parameter in nonlinear fiber optics because it causes the stretching of the pulse duration. Absorption coefficient as a function of wavelength of ABH160 as well as refractive index was replotted as a function of wavelength and shown in fig. (5). This shows that refractive index (absorption coefficient) almost decays exponentially with wavelength of the light.

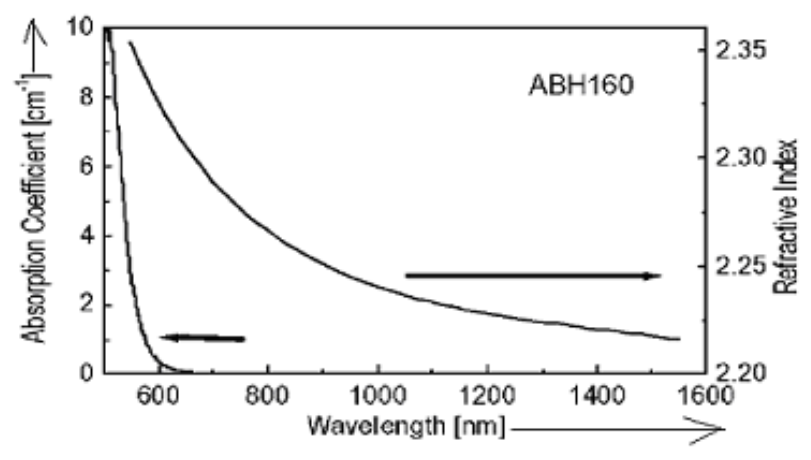

Fig. 5: Absorption coefficient as a function of wavelength of $\mathrm{ABH} 160$ [6]

In optical fiber the total group velocity dispersion is divided into the material dispersion $D_{M}$ and waveguide dispersion $D_{W}$ and $D_{T O T}=D_{M}+D_{W}$, $D_{M}$ and $D_{W}$ for Bi-NLF are given by

$$
\begin{aligned}
& D_{M}=\frac{\lambda}{c} \frac{d^{2} n(\lambda)}{d \lambda^{2}} \\
& D_{W}=-\frac{v n_{2} \Delta}{\lambda c} \frac{d^{2}(v b)}{d v^{2}}
\end{aligned}
$$

Where $\mathrm{c}$ is velocity of light in vacuum $v$ is normalized frequency, $\Delta$ and $\mathrm{b}$ are relative refractive index difference and normalized propagation constant respectively.

Theoretically values of $D_{M}$ of $\mathrm{ABH} 160$ at $1550 \mathrm{~nm}, D_{W}$ of Bi-NLF and $D_{\text {TOT }}$ for ABH160 at $1550 \mathrm{~nm}$ were calculated as $-206 \mathrm{ps} / \mathrm{nm} / \mathrm{km}$, $89 \mathrm{ps} / \mathrm{nm} / \mathrm{km}$ and $-295 \mathrm{ps} / \mathrm{nm} / \mathrm{km}$ respectively and by using homodyne interferometeric method experimental value of total dispersion at $1550 \mathrm{~nm}$ was $-280 \mathrm{ps} / \mathrm{nm} / \mathrm{km}$ which shows a good agreement with the calculated result.

The calculated value of total group velocity dispersion for ABH160 is significantly larger than that of conventional silicate fiber. The large normal dispersion of Bi-NLF is consistent with the strong wavelength dependence of refractive index and small core diameter though total group velocity dispersion in short length $\mathrm{Bi}_{2} \mathrm{O}_{3}$ based fiber is suppressed comparably with $\mathrm{SiO}_{2}$-fibers.

$\mathrm{ABH} 160$ that is $\mathrm{Bi}_{2} \mathrm{O}_{3}$ - based glass was developed as a core medium of Bi-NLF. The 65.5 mole \% $\mathrm{Bi}_{2} \mathrm{O}_{3}$ concentration of $\mathrm{ABH} 160$ almost corresponds to the max $\mathrm{Bi}_{2} \mathrm{O}_{3}$ concentration for the stable glass formation.

Present researchers developed a highly nonlinear fiber made up of the $\mathrm{Bi}_{2} \mathrm{O}_{3}$-based glass (Bi-fiber) and studied its non-linear optical properties.

Four-Wave Mixing Experiment Results and Discussions:

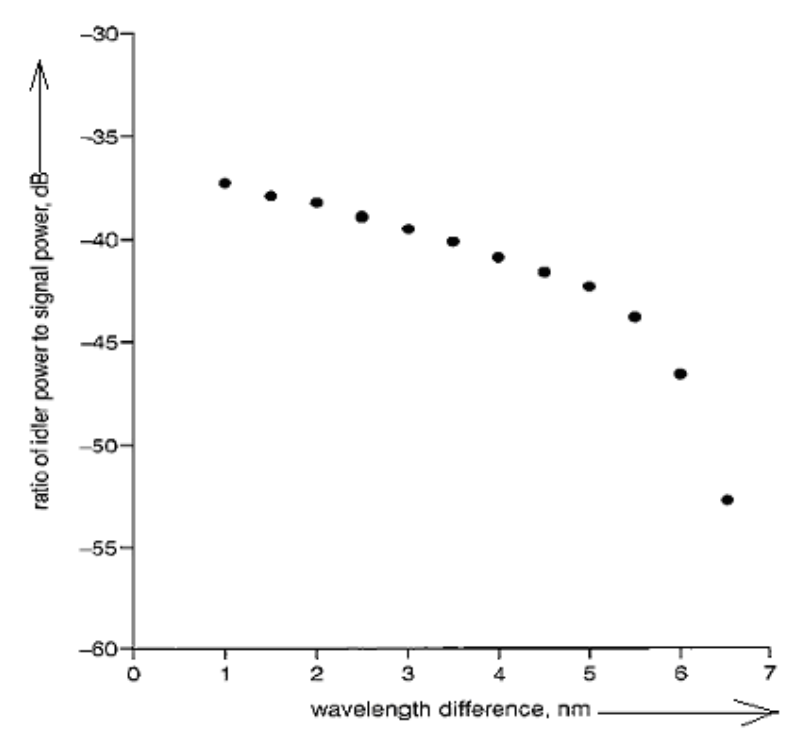

Fig. 6: Variation of ratio of idler power to signal ratio power as function of signal wavelength [7] 
Now newly generated wavelengths are $.8 \mathrm{~nm}$ apart and they all carry the switched data. Researchers used a thin filter with band width $0.6 \mathrm{~nm}$ to filter out switched signal. The output was observed by using an analyzer with $0.01 \mathrm{~nm}$ resolutions and a $50 \mathrm{GHz}$ sampling oscilloscope.

The control signal has an ON/OFF duration of 64 ns. The portion of data signal, which coincides with ON duration of the control signal, will be switched and wavelength converted by the FWM process, where as the portion of the data signal which coincides with OFF period will be blocked. Thus the ON-OFF switch therefore can potentially operate at speed beyond $10 \mathrm{Gbit} / \mathrm{s}[6,7,9]$.

\section{CONCLUSIONS}

I came to conclusions that there was wavelength dependence of refractive index in two types of $\mathrm{Bi}_{2} \mathrm{O}_{3}$ based glasses $\mathrm{ABH} 160$ and $\mathrm{B} 027$. It was studied that how the wavelength dependence of material dispersion of $\mathrm{ABH} 160 \quad\left(D_{M}\right)$ and waveguide dispersion $\left(D_{W}\right)$ of the $\left.\mathrm{Bi}_{2} \mathrm{O}_{3}\right)$ based optical fiber was calculated from the experimentally obtained refractive indices and their wavelength dependence. It was found that the numerical calculations agreed with the experimental values. As an application of $\mathrm{Bi}$ HNLF, the working of proposed all-optical ONOFF switching was described. Refractive index of $\mathrm{ABH} 160$ and Bo27 were found to be exponentially decaying as a function of wavelength. Absorption coefficient of ABH160 as a function of wavelength was found to be decaying exponentially. The process of four wave mixing and consequences was reviewed. Theoretically values of $D_{M}$ of ABH160 at $1550 \mathrm{~nm}, D_{W}$ of Bi-NLF and $D_{\text {TOT }}$ for ABH160 at $1550 \mathrm{~nm}$ were calculated as $-206 \mathrm{ps} / \mathrm{nm} / \mathrm{km}$, $89 \mathrm{ps} / \mathrm{nm} / \mathrm{km}$ and $-295 \mathrm{ps} / \mathrm{nm} / \mathrm{km}$ respectively and by using homodyne interferometric method experimental value of total dispersion at $1550 \mathrm{~nm}$ was $-280 \mathrm{ps} / \mathrm{nm} / \mathrm{km}$ which shows a good agreement with the calculated result.

\section{REFERENCES}

[1] Hasegawa, T.; Nagashima T. and Sugimoto, N. Dispersion properties of $\mathrm{Bi}_{2} \mathrm{O}_{3}$ based high nonlinear optical fiber, $J$. of the Ceramic society of Japan, 114(2): 224-226 (2006).

[2] Can-Uc, Lopez J.; Lizarraga.-MEDINA E. G.; Borron-Nunez, Rangel-Rojo R.; Marquez H.; Tiznado H.; Jurado-Gonzalez J. A. and Hirata-
Flores G. Third-order nonlinear optical properties of a multi-layer $\mathrm{A} 12 \mathrm{O} 3 / \mathrm{ZnO}$ for nonlinear optical waveguides Optics Express 27: 12-17359 (2019).

[3] Liaros N. and Fourkas T. The characterization of Absorptive Nonlinearities Laser Photonics Rev. 11: 1700106 (2017).

[4] Xiang, L. Z.; Yu, H. Z.; Tao, H.; Ci, Z. T. and Lin, X. Z. Study on establishing and testing for strain transfer model of distributed optical fiber senser in concrete structures Optical fiber technology 61: 102430 (2021).

[5] Kikuchi, K.; Taira, K. and Sugimoto. Highly nonlinear Bismuth oxide-based glass fiber for alloptical signal processing, Electronic letter , 38: 4 (2002).

[6] Quereshi, K. K.; Wai, P. K. A.; Tam, H. Y.; Chao, L. and Sugimoto, N. All-optical ONN-OFF Switch based on Bi based highly nonlinear fiber, Microwave and optical technology Letters, 49 (4): (2007).

[7] Gomes, A. S. L. et al. Third order nonlinear optical properties of Bismuth borate glasses measured by conventional and thermally managed eclipse z-scan, J. of Applied Physics, 101: 033-115 (2007).

[8] Boyd, R.W. Nonlinear Optics, London, Academic press (1992).

[9] Juliet T. G. et al. High-nonlinear Bismuth oxide fiber for smooth Super continuum generation at 1.5 micrometer, optics Express 5697. 12: 23 (2004). 